\title{
Kosterlitz-Thouless vs Ginzburg-Landau description of 2D superconducting fluctuations
}

\author{
L. Benfatto, A. Perali, C. Castellani, and M. Grilli \\ Dipartimento di Fisica, Università di Roma "La Sapienza" and \\ Istituto Nazionale Fisica della Materia, Unità di Roma 1, \\ P.le A. Moro, 2 - 00185 - Roma, Italy
}

(July 31, 2018)

\begin{abstract}
We evaluate the charge and spin susceptibilities of the 2D attractive Hubbard model and we compare our results with Montecarlo simulations on the same model. We discuss the possibility to include topological Kosterlitz-Thouless superconducting fluctuations in a standard perturbative approach substituting in the fluctuation propagator the Ginzburg-Landau correlation length with the Kosterlitz-Thouless correlation length.

PACS numbers:74.20.De, 74.20.Mn, 71.10.-w
\end{abstract}

The discovery of spin and charge pseudogaps in the normal state of underdoped superconducting cuprates [1] has triggered a renewed interest on the physics of preformed Cooper pairs. The actual source of the pseudogaps (pairing, and/or spin-, and/or charge fluctuations) and the leading mechanisms responsible for the reduction of the superfluid density at low temperature (classical phase fluctuations, collective modes, quasiparticle excitations) are still debated. However, many indications support the idea that pairing occurs below some crossover temperature $T^{*}$, while the phase coherence is established at a sizable lower temperature. The low density of carriers resulting in a low superfluid density and the short coherence length $\xi_{0} \sim 10 \div 20 \AA$, support the relevance of the superconducting phase fluctuations in the thermodynamic and dynamic properties of these materials. Moreover, although no discontinuity of the superfluid density at $T_{c}$ is observed, the strong anisotropy of the cuprates suggests that some features of a KosterlitzThouless (KT) transition could be present in these systems [2]. Therefore it is worth investigating the effects of the topological vortex-antivortex phase fluctuations on the various properties of a $2 \mathrm{D}$ superconductor. In particular, an important issue concerns the inclusion of these effects in evaluating thermodynamic quantities like the spin susceptibility or the charge susceptibility. In this context, the aim of the present work is to look for possible connections between the perturbative scheme leading to the standard time-dependent Ginzburg-Landau (TDGL) results and the KT physics.

Halperin and Nelson [3] have shown that, in the KT regime, the contributions of superconducting fluctuations to the conductivity above $T_{K T}$ have the same functional form, in terms of the correlation length $\xi$, as the Aslamazov-Larkin contributions of the standard TDGL theory, $\sigma_{K T}(\xi) \simeq \sigma_{G L}(\xi) \sim \xi^{2}$. The same holds for the fluctuation contribution to the diamagnetism $\chi_{K T}^{d}(\xi) \simeq$ $\chi_{G L}^{d}(\xi) \sim \xi^{2}$. In spite of the same correlation length dependence, conductivity and diamagnetism in KT or
TDGL theory have a completely different temperature dependence, induced by the different temperature dependence of the correlation length in the two theories. The KT correlation length diverges exponentially at $T_{K T}$ while the GL correlation length diverges as a power-law with the classical exponent $\nu=\frac{1}{2}$. Therefore the KT conductivity and the diamagnetic susceptibility diverge exponentially at $T_{K T}$ while the same quantities in the TDGL theory diverge as a power-law at $T_{c}$ with a critical exponent $\gamma=1$. In the present work we investigate the possibility that, in analogy with conductivity and diamagnetism, the correct behavior of the spin and charge susceptibilities in the KT regime can be simply recovered by inserting the KT correlation length in their TDGL expressions. We shall find that this prescription does work for the spin susceptibility while it does not for the charge susceptibility.

We analyze the two-dimensional negative- $U$ Hubbard model [10] which is the simplest minimal model where the distinct occurrence of pairing and phase coherence can be investigated. Within this model, the spin susceptibility $\chi_{s}$ and the charge compressibility $\chi_{c}$ are calculated on a two-dimensional square lattice by performing a loop expansion with the fermions exchanging the Cooperfluctuations propagator in the standard form. Before giving the technical details of our treatment, we immediately present our results.

Figure 1 shows the behavior of the spin susceptibility when the correlation length is assumed either of the GL form (dashed line with crosses) or of the KT form (dotted line with stars). Both curves are compared with the Montecarlo data obtained in Ref. [6,7] for the negative- $U$ Hubbard model with $U=-4 t$ ( $t$ is the nearest-neighbor hopping) at filling $n=0.5$ electrons per cell. The critical temperature $T_{K T}$ of the KT superconducting transition, as extracted from numerical calculations, is $T_{K T}=0.05 t$ and has been used as the input critical temperature for our perturbative calculations. In the Montecarlo data, for $T$ less then $T^{\star} \simeq t \gg T_{K T}, \chi_{s}$ starts decreasing. This 
indicates the existence of strong superconducting fluctuations in the temperature range between the mean-field transition temperature $\left(T_{B C S} \simeq 0.6 t\right)$ and the true $\mathrm{KT}$ transition. It is apparent from Fig. 1 that the rapid decrease of the spin susceptibility in the Montecarlo results is well fitted by inserting in the correlation length the KT temperature dependence as given by the expression

$$
\xi_{K T}(T)=\xi_{c} \exp \left[b \sqrt{\frac{T\left(T_{B C S}-T_{K T}\right)}{T_{B C S}\left(T-T_{K T}\right)}}\right] .
$$

Here $\xi_{c}$ is an effective size of the core of the vortex that we take of the order of the zero temperature correlation length $\xi_{0}$, and $b$ is a positive constant of the order of unity. This specific form of the KT correlation length has been derived along the line of Ref. [3], although it differs slightly from the one commonly quoted in the literature [1,5]. We shall comment on this later. Notice that the KT mass term (inverse square of the correlation length) of the Cooper propagator remains small and generates strong fluctuations, in a wider range of temperatures than the GL mass with the same critical temperature in agreement with Montecarlo data. The GL correlation length is instead completely inadequate to reproduce the Montecarlo data in the all range of temperatures.

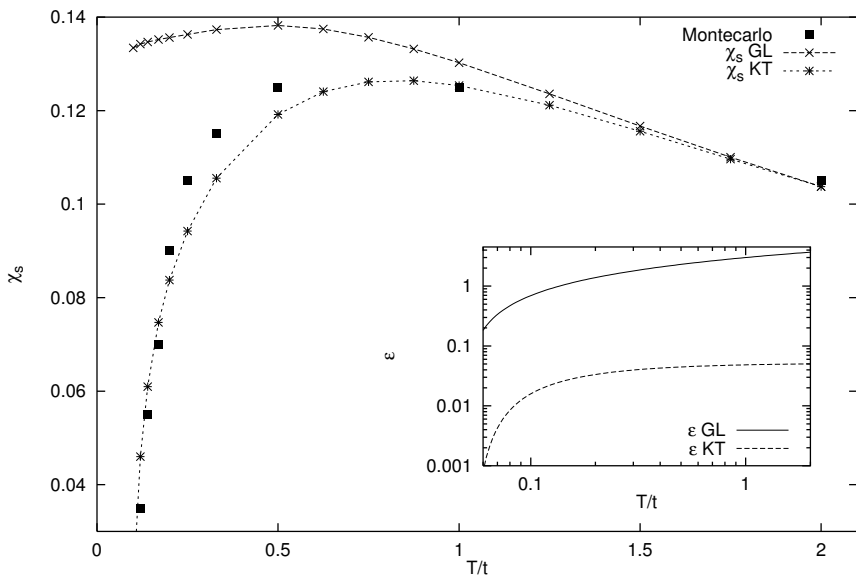

FIG. 1. Comparison between Montecarlo spin susceptibility (taken from Ref. [6]) and the spin susceptibility calculated using the Ginzburg-Landau $\left(\chi_{s}^{G L}\right)$ and the Kosterlitz-Thouless $\left(\chi_{s}^{K T}\right)$ correlation length.

The fit in Fig. 1 stops at $T \simeq 0.1 t$ because there are no numerical data below this value. This also appears to be the lower limit for our approach to work. Indeed for $T \simeq 0.09 t$ the TDGL expression for $\chi_{s}$ develops a non physical behavior $\left(\chi_{s}<0\right)$, indicating that the perturbative scheme no longer applies near $T_{K T}$. Whit this caution in mind, the results of Fig. 1 indicate that the simple loop expansion we adopted is able to reproduce the spin susceptibility in a wide range of temperatures. They support the idea that the main effect of the vortex-antivortex phase fluctuations on the spin susceptibility is embedded in (and satisfactorily accounted for by) the temperature dependence of the $\xi_{K T}(T)$ correlation length, in analogy with the conductivity and diamagnetism.

On the other hand, as seen in Fig. 2, the same type of calculations for the charge susceptibility fail in describing the nearly constant (but with sizeable error bars) behavior obtained numerically. In particular, we find that the Aslamazov-Larkin (AL) contribution, which does not contribute to the spin susceptibility, strongly enhances $\chi_{c}(T)$ and eventually leads to a divergent $\chi_{c}$ near $T_{K T}$. As a consequence $\chi_{c}(T)$ strongly deviates from the Montecarlo results for $T<T_{B C S}$. In Fig. 2 we also report the RPA resummation of the bare bubble in the charge channel that fits the available Montecarlo data, to obtain, by extrapolation, the $\chi_{c}(T)$ at higher temperature.

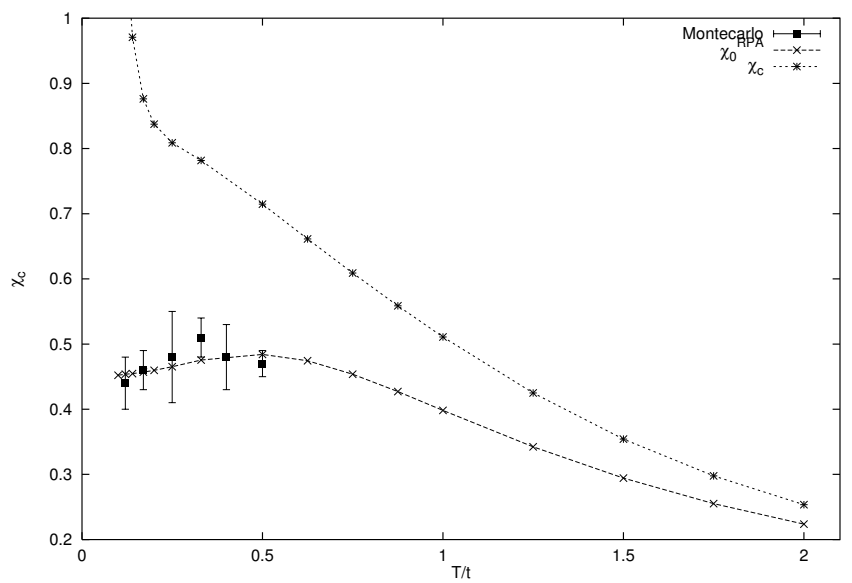

FIG. 2. Comparison between Montecarlo charge susceptibility (taken from Ref. [8]), the charge susceptibility calculated using the Kosterlitz-Thouless correlation length $\left(\chi_{c}\right)$ and the RPA resummation of the bare bubble $\left(\chi_{0}^{R P A}\right)$.

With this respect $\chi_{c}$ appears to behave as the specific heat $c_{v}$, for which the 2D-TDGL expression $c_{v} \sim \xi_{G L}^{2}$ does not reproduce the correct $\mathrm{KT}$ result $c_{v} \sim \xi_{K T}^{-2}$, even when expressed in terms of the correlation length. For the specific heat this happens despite the free energies in the two theories have the same leading behavior when written in terms of the respective correlation lengths: $F_{G L} \sim \xi_{G L}^{-2} \ln \xi_{G L}$ and $F_{K T} \sim \xi_{K T}^{-2}$, since $c_{v}$ involves the second derivative of $F$ with respect to temperature the different temperature dependences of the correlation lengths (and the subleading $\ln \xi_{G L}$ factor) lead to completely different results in the two theories. Our result for $\chi_{c}$ has the same origin: The charge response at $\omega=0, q \rightarrow 0$ can be obtained as a chemical potential derivative of the free energy. Now, since the critical temperature depends on the chemical potential $T_{c}=T_{c}(\mu)$, a total derivative with respect to $\mu$ also involves derivatives with respect to $T_{c}$, and, in turn, derivatives of $\xi$. Therefore the temperature dependence in $\chi_{c}$ 
not only arises from the temperature dependence of $\xi(T)$, but also depends on $d \xi / d T_{c}$. In fact one gets the same TDGL singular contribution $\sim \xi^{2}$ for $\chi_{c}$ and $c_{v}$. Our simple perturbative expansion, where the leading temperature dependence only arises from the mass term $\xi^{-2}$ of the Cooper fluctuation propagator in the TDGL expression, fails to reproduce the correct temperature dependence for $\chi_{c}$ in the same way as it fails in evaluating the specific heat.

We now describe the details of our calculations. The model we consider is given by

$$
H=-t \sum_{<i, j>\sigma} c_{i \sigma}^{\dagger} c_{j \sigma}+U \sum_{i} n_{i \uparrow} n_{i \downarrow}-\mu \sum_{i \sigma} n_{i \sigma}
$$

where $t$ is the hopping between nearest-neighbor sites, $U<0$ the strength of the attraction and $\mu$ the chemical potential. The standard ladder resummation of diagrams leads to the Cooper pair propagator $L\left(q, \Omega_{l}\right)=-U /\left(1+U \chi_{0}^{p p}\left(q, \Omega_{l}\right)\right)$ where $\chi_{0}^{p p}\left(q, \Omega_{l}\right)$ is the bare particle-particle bubble, being $q$ the momenta and $\Omega_{l}$ the Matsubara frequency. In the normal state, within the standard GL approach, at small $q$ and $\Omega_{l}$ one has

$$
L^{-1}\left(q, \Omega_{l}\right)=N_{0}\left(\epsilon+\eta q^{2}+\gamma\left|\Omega_{l}\right|\right)
$$

where $N_{0}$ is the density of states at the Fermi energy, $\eta=7 \zeta(3) /\left(32 \pi^{2}\right)\left(v_{F} / T_{c}\right)^{2} \simeq \xi_{0}^{2}$, and $\gamma=\pi /\left(8 T_{c}\right)$. The mass term $\epsilon=\ln \left(T / T_{c}\right)=\left(\xi / \xi_{0}\right)^{-2}$ of the propagator controls the distance from the superconducting transition. In the standard GL approach $\epsilon \sim \xi_{G L}^{-2}$ and near $T_{c}$ it goes to zero as $\left(T-T_{c}\right) / T_{c}$.

We study the charge and spin susceptibilities by evaluating the one loop corrections $\Delta \chi_{c}$ (charge channel) and $\Delta \chi_{s}$ (spin channel) to the bare particle-hole bubble $\chi_{0}^{p h}, \chi_{c, s}^{p h}=\chi_{0}^{p h}+\Delta \chi_{c, s}$. The charge $(c)$ and spin (s) bubbles $\chi_{c, s}^{p h}$ are then inserted in the RPA resummation to get the charge and spin susceptibilities (see below). In the one loop expansion, we include diagrams containing only one integration on the bosonic variables $\left(q, \Omega_{l}\right)$ (i.e. one bosonic loop) of the fluctuation propagator $L\left(q, \Omega_{l}\right)$, obtaining three kinds of diagrams which contribute differently to the spin and charge susceptibilities: the selfenergy diagrams, where $L\left(q, \Omega_{l}\right)$ renormalizes the one particle bare Green function (DOS contribution); the vertex diagrams, where $L\left(q, \Omega_{l}\right)$ renormalizes the vertex, connecting two bare Green function (MakiThompson (MT) contribution); the Aslamazov-Larkin (AL) diagrams, containing two fluctuation propagators. Moreover it is necessary to add the counterterms (CT) proportional to the shift of the chemical potential $\delta \mu$, which is required to preserve the number of particles. We notice that the one loop expansion for the charge and the spin susceptibilities satisfies the relation, derived from spin and charge conservation, $\chi_{s, c}(q=0, \Omega \neq 0)=0$. One obtains:

$$
\begin{aligned}
& \Delta \chi_{s}=4 \cdot D O S-2 \cdot M T+4 \cdot C T \\
& \Delta \chi_{c}=4 \cdot D O S+2 \cdot M T+4 \cdot A L+4 \cdot C T .
\end{aligned}
$$

The absence of the AL contribution and the (opposite) sign of the MT diagrams in the spin susceptibility is the consequence of the vertex spin structure, as shown in Ref. 11. Moreover the leading DOS contributions to the charge susceptibility cancel the MT ones. The AL diagrams give therefore the most important contribution to the charge susceptibility (being the CT diagrams subdominant respect to them) [12].

According to the physical assumption outlined above that the TDGL and KT temperature dependencies are essentially ruled by the correlation lengths, we have alternatively taken Eq.(3) with $\xi=\xi_{G L}$ and $\xi=\xi_{K T}$. In the calculation with $\xi_{G L}$ we used $T_{c}=T_{K T}$ and the mass term $\epsilon=\ln \left(T / T_{c}\right)$, while in the calculation with $\xi_{K T}$ we used Eq.(11) with $b=1.6$ and $\xi_{c}=\xi_{0}$. In both cases we took the coefficients $\eta$ and $\gamma$ given by the corresponding expressions reported below Eq.(3) calculated with $T_{c}=T_{B C S}$. This choice was motivated by the plausible assumption that $\eta$ and $\gamma$ change little once the fluctuations are predominantly in the phase sector. In any case we checked that our results are rather stable with respect to modifications of $\eta$ and $\gamma$.

The charge and the spin susceptibilities are finally obtained by the RPA resummation of the corrected charge and spin bubbles $\chi_{c, s}=\chi_{c, s}^{p h} /\left(1 \pm\left(\tilde{U}_{c, s} / 2\right) \chi_{c, s}^{p h}\right)$ where the plus (minus) sign is associated to the charge (spin) susceptibility. Notice that, following the analysis of Ref. [6], the RPA expressions of both susceptibilities contain an effective local interaction $\tilde{U}_{c, s}$ instead of the bare $U$ in order to properly fit the high temperature region of the Montecarlo data. The validity of the RPA form for the spin susceptibility is also found in the context of the positive- $U$ Hubbard model [13]. However, while in Ref. [6] the bare bubbles were resummed and a value $\tilde{U}=6.5$ was obtained for $U=-4 t$ and $\langle n\rangle=0.5$, in our case we resum the bubbles already containing the $\Delta \chi_{s}$ corrections and a different value $\tilde{U}_{s}=-4.6$ is needed to match the RPA calculation with the high temperature Montecarlo data. For the charge susceptibility the comparison with the RPA resummation in terms of the $\chi_{0}^{p h}$ reported in Fig. 2 gives $\tilde{U}_{c}=-1.6$.

We now comment on the expression in Eq.1 that we used for the KT correlation lenght. We wrote this expression following Halperin and Nelson [3]. They introduce into the $\mathrm{KT}$ correlation length $\xi_{K T} \simeq$ $a \exp \left[b\left(\pi J / k_{B} T-1\right)\right]$ for the classical XY model (with coupling $\mathrm{J}$ and lattice spacing $a$ ) a temperature dependent $J(T)=n_{s}(T) / 8 m$ and take $a=\xi_{c}$. Here the superfluid density $n_{s}(T)$ is taken to vanish linearly at a temperature $T_{0}\left(>T_{K T}\right)$ to be determined selfconsistently by the request that $T_{0}$ should include the effect of the fluctuations at scale lower than $\xi_{c}$. Our expression (1) is 
obtained by taking $T_{0} \simeq T_{B C S}$ and $\xi_{c} \simeq \xi_{0}$ with the idea that phase fluctuations are the most important effect all over the range of temperatures $T_{K T} \lesssim T \lesssim T_{B C S}$ (at least in evaluating $\chi_{s}$ and $\chi_{c}$ ) 14.

The results of the simple procedure outlined above are quite satisfactory for the spin susceptibility. This indicates that the main temperature dependence of this quantity actually arises from the specific KT temperature dependence of the correlation length, which thus brings along the physics of the vortex-antivortex phase fluctuations into a simple perturbative scheme. The same is not true for the compressibility, as for the specific heat, since these quantities also involve temperature derivatives of $\xi_{K T}$.

Our method, developed for the 2D attractive Hubbard model, can be useful to understand the role of the superconducting phase fluctuations in quasi-2D cuprate superconductors. In this context the recent finding that $\mathrm{KT}$ signatures, which are absent in the static conductivity, are progressively more evident in the dynamical conductivity at shorter timescales [2] encourages to extend our analisys to other frequency-dependent quantities. In particular it is of obvious interest to explore the possibility to include in a simple perturbative scheme along the lines followed in the present work the effects of KT topological phase fluctuations on dynamical quantities like the optical conductivity and single-particle spectra.

Acknowledgments. We acknowledge S. Caprara, C. Di Castro, P. Pieri, G. C. Strinati and A. A. Varlamov for helpful discussions.
[1] For a recent review see, e.g. T. Timusk and B. Statt, Rep. Prog. Phys. 62, 61 (1999).

[2] J. Corson, R. Mallozzi, J. Orenstein, J. N. Eckstein, and I. Bozovic, Nature 398, 221 (1999).

[3] B. I. Halperin and D. R. Nelson, J. of Low T. Phys. 36, 599 (1979).

[4] J. M. Kosterlitz and D. J. Thouless, J. Phys. C 6, 1181 (1973).

[5] J. M. Kosterlitz, J. Phys. C 7, 1046 (1974). For a recent review see, Z. Gulácsi and M. Gulácsi, Advances in Physics 47, 1 (1998).

[6] M. Randeria et al., Phys. Rev. Lett. 69, 2001 (1992).

[7] J. M. Singer et al., Phys. Rev. B 54, 1286 (1996).

[8] N. Trivedi and M. Randeria, Phys. Rev. Lett. 75, 312 (1995).

[9] We have consider a sligthly modified propagator which has the periodicity of the lattice, substituting the $q^{2}$ term with $2\left(2-\cos q_{x}-\cos q_{y}\right)$; for small $q$ the two expressions are equivalent.

[10] For a review see, e.g. R. Micnas, J. Ranninger, and S. Robaszkiewicz, Rev. Mod. Phys. 62, 113 (1990).

[11] A. A. Varlamov, G. Balestrino, E. Milani, D. V. Livanov, Advances in Physics, 48, 6, 1 (1999).

[12] Within an expansion of the bare density of states with respect to the energy, the AL contribution is proportional to $\left(1 / N(\mu) \cdot d N(E) /\left.d E\right|_{E=\mu}\right)^{2}$. This is in agreement with the above discussion relating the $\mathrm{AL}$ contribution and $\chi_{c}$ to the dependence of the critical temperature on $\mu$.

[13] N. Bulut et al., Physica C 246, 85 (1995).

[14] Our expression is at variance with respect to the one obtained in Ref. [15] within a T-matrix selfconsistent approach. Their $\xi_{K T}$, rather strangely, would be obtained, in the context of Halperin and Nelson analysis, by assuming a linear increasing temperature dependence of the superfluid density for $T>T_{K T}$.

[15] J. R. Engelbrecht and A. Nazarenko, cond-mat/9806231, preprint (1998). 